\title{
A Case of Infarcted Localized Tenosynovial Giant Cell Tumor of the Knee: Multimodality Imaging Features and Literature Review
}

\author{
Wonju Hong ${ }^{1}$, Sun-Young Park ${ }^{1, *}$, Soo Kee Min ${ }^{2}$, Je-Hyun Yoo ${ }^{3}$, Sung Hye Koh ${ }^{1}$ and Kwanseop Lee \\ ${ }^{1}$ Department of Radiology, Hallym University Sacred Heart Hospital, Anyang, Korea \\ ${ }^{2}$ Department of Pathology, Hallym University Sacred Heart Hospital, Anyang, Korea \\ ${ }^{3}$ Department of Orthopedic Surgery, Hallym University Sacred Heart Hospital, Anyang, Korea \\ "Corresponding author: Department of Radiology, Hallym University Sacred Heart Hospital, Anyang, Korea. Tel: +82-313805969, Email: sunypark83@naver.com
}

Received 2018 March 19; Revised 2018 October 14; Accepted 2018 October 16.

\begin{abstract}
Localized tenosynovial giant cell tumor (L-TGCT), formerly called giant cell tumor of the tendon sheath, is one of the benign proliferative diseases of the synovium of a joint, bursa, or tendon sheath. L-TGCT occasionally involves a large joint such as the knee, and rarely it is combined with infarction by pedicle torsion. Neither the prevalence of torsion of L-TGCT nor the mechanism involved are known, but it is thought to be uncommon progress. Herein, we describe a rare case of infarcted L-TGCT in the knee involving torsion of a mass pedicle in a 47-year-old man. We utilized magnetic resonance imaging including diffusion-weighted imaging and axial apparent diffusion mapping, and sonographic appearance during the course of investigations, because radiologic findings showed characteristic features of infarcted L-TGCT.
\end{abstract}

Keywords: Infarction, Knee Joint, Magnetic Resonance Imaging, Tenosynovial Giant Cell Tumor, Ultrasound

\section{Introduction}

Tenosynovial giant cell tumor (TGCT) is a family of benign proliferative diseases of the synovium of a joint, bursa, or tendon sheath (1). TGCTs are usually divided according to their site (intra-articular or extra-articular) and growth pattern (localized or diffuse), and such differences are usually associated with differences in their clinical features and biological behavior, though they appear to share some common aspects of pathogenesis (2). Localized forms predominate in the knee, and other locations include the wrist, foot, ankle, and hand. Localized intraarticular TGCT frequently involves the knee, and the common location is reportedly the infrapatellar area $(2,3)$. Patients may present with slowly progressing pain, swelling, decreased range of motion, or a painless mass.

A few cases of infarcted localized tenosynovial giant cell tumor (L-TGCT) have been described in the orthopedic literature (4-7), and in these cases the patients complained of acute symptoms including sudden-onset pain or locking. All these lesions had undergone infarction and exhibited pedicle torsion arising from the synovial capsule on arthroscopy. There have been only three reported patients with histologically and radiologically confirmed infarcted L-TGCT in the knee or hip $(4,5,7)$. To our knowledge, the current report is the first to describe a case of this entity using a combination of radiographic, sonographic, and magnetic resonance imaging including diffusion-weighted imaging (DWI) and axial apparent diffusion coefficient (ADC) mapping, in conjunction with arthroscopic and histological findings. The details of this case will be helpful when interpreting radiologic findings of a solitary nodule in the knee that raise the possibility of this rare entity, L-TGCT.

\section{Case Presentation}

A 47-year-old man presented with a painless mass in the right knee that had been there for more than 1 year. The mass was located in the superior portion of the patella. It was non-tender, soft, round, and mobile, but caused mass effect at the knee joint during deep flexion. Radiological examinations were performed to characterize and diagnose the soft tissue mass.

Initial radiographs of the right knee yielded no remarkable findings, but lateral radiography revealed fullness of the suprapatellar pouch. On ultrasound (US), an approximately $2.8 \times 1.3$-cm ovoid mass with well-delineated margins was located at the suprapatellar area of the right knee. It exhibited heterogeneous echogenicity. Color Doppler investigations revealed no vascularity (Figure $1 \mathrm{~A}$ ). 
There was a small to moderate amount of joint effusion with diffuse synovial thickening at the suprapatellar pouch, suggesting underlying mild synovitis. Conventional magnetic resonance imaging(MRI) yielded the same morphologic findings as US with characteristic signal intensities. The lesion showed inhomogeneous intermediate to high signal intensity on T1-weighted imaging (Figure 1B). On T2-weighted imaging, the mass showed inhomogeneous high signal intensity compared to skeletal muscle (Figure 1C). It was not suppressed on fat-suprresed imaging (Figure 1D). The mass was surrounded by a thin rimlike structure with an even thickness that exhibited the same signal intensity as synovium on all sequences, and it also had a pedicle arising from the lateral side of the joint capsule, anterior to the lateral femoral condyle (arrows in Figure $1 \mathrm{C}$ and $\mathrm{D}$ ). The mass exhibited either subtle enhancement or no definite enhancement on contrastenhanced T1-weighted fat suppressed imaging (Figure 1E) (it was not easy to evaluate precisely, because there was no T1 fat-suppressed image). The synovium adjacent to the lesion at the suprapatellar pouch exhibited proliferation with diffuse thick enhancement with a small amount of joint effusion (Figure 1E). No meniscal tear or chondromalacia was identified.

Axial DWI (TR/TE 8110/64) showed increased signal intensity of the entire mass and a rim with dark signal intensity(Figure1F). ADC mapping of the suprapatellar mass was $1686.80 \mathrm{~mm}^{2} / \mathrm{s}\left(\mathrm{b}=0,400\right.$, and $\left.800 \mathrm{~mm}^{2} / \mathrm{s}\right)$ (Figure $1 \mathrm{G}$ ).

The patient underwent arthroscopic excision of the mass. There was a 3.5-cm long, ellipsoid-shaped, wellmarginated mass with a pedicle originating from the synovial capsule in a suprapatellar pouch. This mass appeared to be encapsulated with synovium.

With regard to gross pathology, the soft tissue mass was pink, and included a necrotic portion on the cut surface (Figure 2A). Histologically, the lesion had a stalk which was connected to synovial lining, and it was surrounded by collagenous tissue. Most of the lesion was infarcted, thus the nuclei of most cells had disappeared (Figure 2B). It was difficult to distinguish the origins of the cells in the infarcted portion. Nonetheless, in the viable area microscopy revealed multinucleated giant cells, macrophages, foam cells, and fibroblasts. There was hemosiderin pigment in macrophages and in the extracellular space. A portion was composed of areas of hemorrhage with red blood cells (Figure $2 \mathrm{C}$ ). It was confirmed as L-TGCT with infarction. We assumed that infarction was caused by torsion of the mass, which could be explained by the pedicle from the synovial capsule.

The patient was followed up for one year and did not have any symptom or recurrence of the tumor during the follow-up period.

\section{Discussion}

TGCTs are now classified as benign, so-called fibrohistiocytic tumors in the World Health Organization system of classification of bone and soft tissue tumors (2). Knees are the joints most commonly affected by TGCTs, and in the localized intra-articular form knees account for $80 \%$ of cases, followed by hip, ankle, shoulder, and elbow (8). The most common location in the knee is known as the infrapatellar area. One study reported that $67 \%$ of the lesions were infrapatellar in location, $24 \%$ suprapatellar, and $10 \%$ posterior intercondylar (5). It affects patients aged 20 - 50 years.

Clinical manifestations of L-TGCT are various. Patients commonly present with gradually progressing pain, swelling, and decreased range of motion, and in some cases a soft tissue mass is evident (3). It is known that in most cases the onset is chronic, with a duration of months to years (9); however, in the cases of infarction we reviewed, all patients complained of acute symptoms within 72 hours including sudden-onset pain or locking (5-7). In the current case, the patient presented with a palpable painless soft tissue mass, but he could not remember if acute pain had been present.

The prevalence of torsion or infarction of L-TGCT remains unknown, as does the mechanism involved, but it is thought to be uncommon progress. Few cases of L-TGCT with torsion or infarction have been reported in the literature (4-7). In 1985, the first orthopedic cases of torsion of L-TGCT were described by Howie et al. (6) in a report in which they described three cases of intra-articular L-TGCT (previously called localized pigmented villonodular synovitis) with evidence of torsion in knee joints. All cases contained areas of infarction with hemorrhage and necrosis as determined histologically. The lesions arose from the medial side of the joint, above the coronary ligaments, the infrapatellar fat pad, and the intercondylar notch. Since then, only three case reports with MRI findings have been published $(5,7)$. Huang et al. (7) reviewed 21 cases of LTGCT (previously called localized nodular synovitis) that included MRI and clinical findings, and 1 patient showed torsion of the mass in an infrapatellar lesion with acute symptoms. They only described findings of a pedicle, no additional imaging features pertaining to the diagnosis of torsion of the mass on MRI, but notably detection of the pedicle can be difficult unless images are carefully inspected. Fukui et al. (5) reported 1 case of intra-articular L-TGCT (previously called localized pigmented villonodular synovitis) of the hip, an uncommon location, with sudden-onset pain caused by torsion of the tumor pedicle. The mass exhibited low signal intensity on T1-weighted images and high signal intensity on T2-weighted images, and marginal enhancement on gadolinium-enhanced im- 

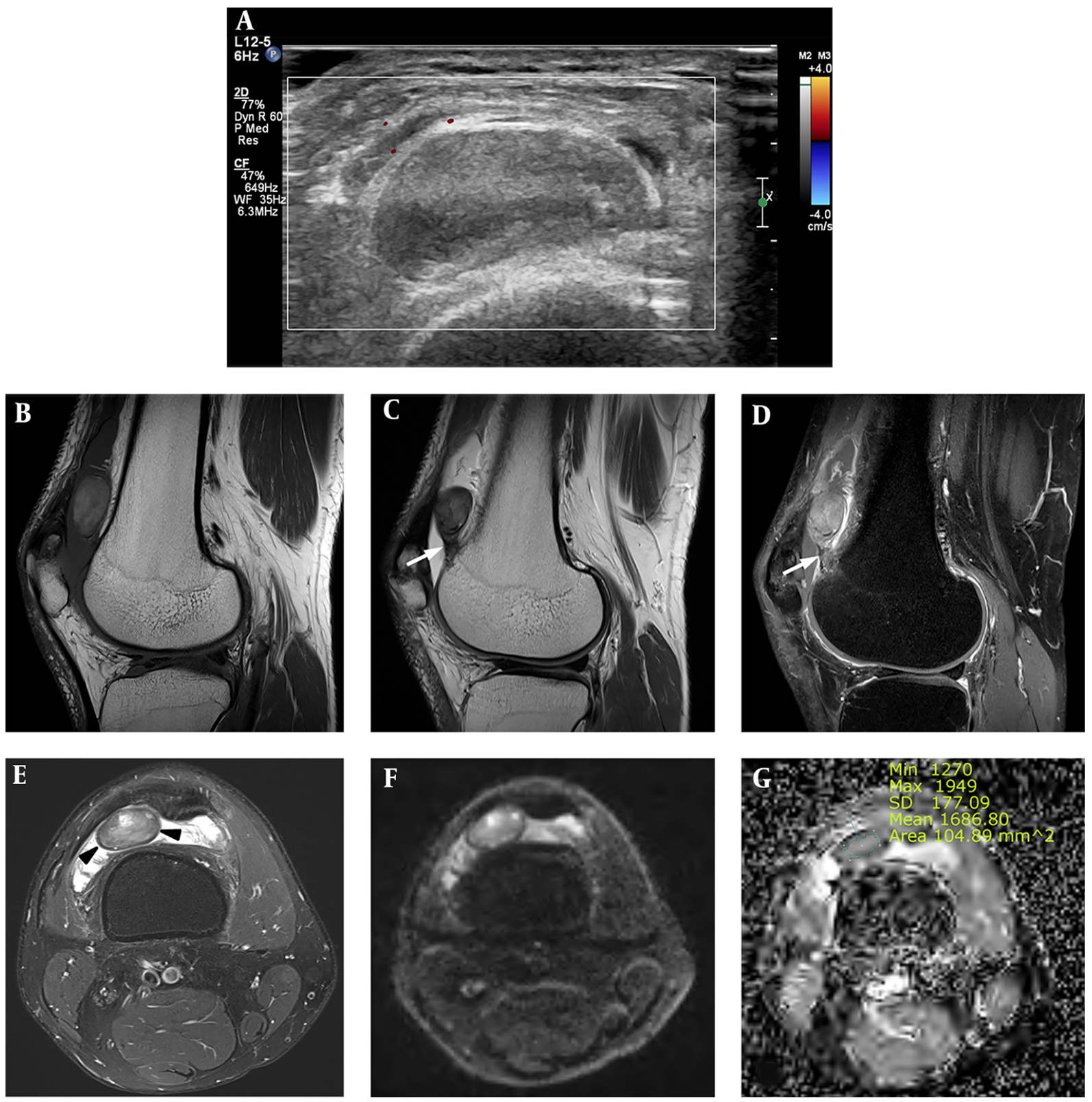

Figure 1. A 47-year-old man with a painless mass in the suprapatellar area of the right knee. An approximately $2.8 \times 1.3$-cm ovoid mass exhibited heterogeneous echogenicity without vascularity on color Doppler image (A). The mass exhibited inhomogeneous intermediate to high signal intensity on T1-weighted imaging (B) and inhomogeneous high signal intensity compared to skeletal muscle on T2-weighted imaging (C). It was not suppressed on fat-suppressed imaging (D). On contrast-enhanced T1-weighted fatsuppressed imaging, the mass exhibited mild enhancement. Adjacent to the lesion at the suprapatellar pouch, the synovium showed proliferation with diffuse thick enhancement with a small amount of joint effusion, suggesting synovitis (E). The mass was surrounded by a thin rim-like structure with an even thickness (arrowheads in E), and had a pedicle (arrows in C and D) arising from the medial side of the joint capsule, anterior to the medial femoral condyle. Axial diffusion-weighted images (repetition time [TR]/echo time [TE], 8110/64) show increased signal intensity of the entire mass, with a rim exhibiting dark signal intensity $\left(\mathrm{b}=800 \mathrm{~mm}^{2} / \mathrm{s}\right)(\mathrm{F})$. Axial apparent diffusion coefficient mapping of the suprapatellar mass was $1686.80 \mathrm{~mm}^{2} / \mathrm{s}(\mathrm{G})$.

ages. Lastly, Cheng et al. (4) described a case of a 36-yearold woman with localized nodular synovitis of the knee with an extensive area of infarction and hemorrhage. MRI revealed a $2-\mathrm{cm}$ well-defined oval mass displacing the in- frapatellar fat pad, exhibiting high signal intensity on T1weighted, T2-weighted, and proton density images. Mild enhancement was noted on gadolinium-enhanced images. The mass was mistaken as intra-articular chondroma or 

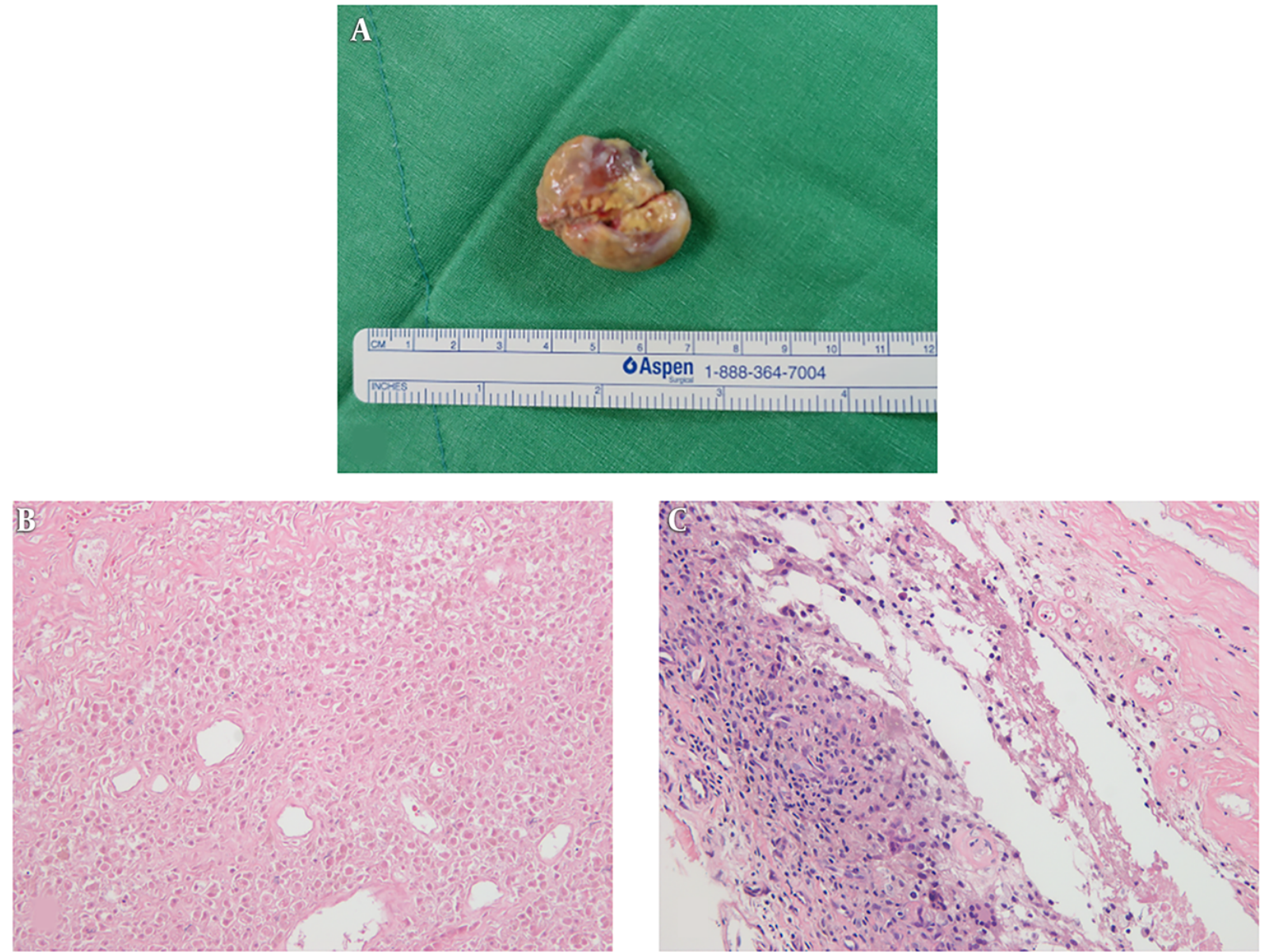

Figure 2. Gross and histologic pathology of the soft tissue mass. With regard to gross pathology, the soft tissue mass was pink, and exhibited a necrotic portion on its surface (A). Most of the lesion was infracted, so the nuclei of cells had disappeared. Some parts were composed of areas of hemorrhage with red blood cells (hematoxylin and eosin staining; original magnification $\times 200$ ) (B). The mass was surrounded by collagenous tissue and synovial lining. In the viable area of the tumor, histology findings included multinucleated giant cells, macrophages, foam cells, and fibroblasts with hemosiderin pigment deposits in macrophages and in the extracellular space (hematoxylin and eosin staining; original magnification $\times 200)(C)$.

osteochondroma, but the pathology was compatible with localized nodular synovitis with a large area of infarction and hemorrhage. Thus, the current case is the fourth known case of infarcted L-TGCT reported in conjunction with radiographic, sonographic, MRI with DWI and ADC mapping, intraoperative, and histological data.

Ultrasound appearances of L-TGCT are usually nonspecific, with the entity depicted as a solitary synovial mass with possible associations with joint effusion or bone erosion (10). Typically, increased vascularity is seen in the synovial mass on Doppler imaging (10). However, the current case was completely avascular on Doppler imaging, which was thought to be caused by hemorrhage and necrosis due to infarction. This also explains why enhancement was very subtle or nearly absent on MRI.

MRI has been reported to be a sensitive modality for identifying features consistent with TGCT, but it is still not specific (11). In most cases the signal intensity is intermediate to low on T1-weighted images, and low signal intensity also predominates on T2-weighted images due to the preferential shortening of $\mathrm{T} 2$ relaxation time caused by hemosiderin (1). Notably however, the current case exhibited higher signal intensities than typical cases on both T1 weighted and T2-weighted images. The high signal intensity on T1-weighted images was perhaps due to hemorrhage, and the focal area of high signal intensity on T2weighted images indicated necrosis.

Diffusion-weighted images and mean ADC values are helpful for differentiation between benign and malignant soft tissue masses (12). Benign tumors tend to exhibit higher ADC values than malignant tumors, though there are some exceptions including TGCTs. TGCTs are known to 
exhibit low ADC values despite being benign lesions (13). In contrast to previous reports, the current case exhibited a high ADC value $\left(1686.80 \mathrm{~mm}^{2} / \mathrm{s}\right)$ with high signal intensity on DWI. This may represent an increase in extracellular water or loss of cell membrane integrity explained by necrotic fluid due to infarction (14). It also supports the previously mentioned imaging features of conventional MRI.

The current case exhibited characteristic imaging features distinct from other solitary nodules of the knee. The torsioned pedicle arising from the lateral side of the joint capsule was clearly visible with continuity of the synovial lining. Therefore, the possibility of a tumor of synovial origin including L-TGCT could be considered most likely, although the mass was located in the suprapatellar pouch with atypical imaging features on MRI. Other nontumorous conditions such as hematoma are potential differential diagnoses, but hematoma does not have a pedicle, its appearance is less nodular, it lacks solid enhancing tissue, and it may present with fluid-fluid level (4).

In summary, when evaluating a soft tissue mass of the knee joint, it is not quite difficult to diagnose L-TGCT via well-known typical imaging findings, showing intermediate to low signal intensities on both T1-weighted and T2-weighted images with enhancement on gadoliniumenhanced images. However, the current infarcted TGCT exhibited higher signal intensities than typical cases of TGCT on both T1-weighted and T2-weighted images and showed completely avascular on Doppler imaging. These findings are due to hemorrhagic and necrotic changes of the mass caused by infarction. The torsioned pedicle of the mass arising from the joint capsule on MRI is characteristic imaging feature which suggests the infarction of the mass caused by torsion. Recognition of theses appearances on MRI and ultrasound enables to make a possible diagnosis of infarcted L-TGCT as a solitary nodule of the knee.

\section{Footnotes}

Authors' Contributions: Study concept and design: Wonju Hong; data acquisition: Sung Hye Koh; data analysis and interpretation, Sun-Young Park; drafting of the manuscript: Wonju Hong; critical revision of the manuscript for important intellectual content: SunYoung Park; administrative, technical, and material support: Sung Hye Koh and Soo Kee Min; study supervision: Kwanseop Lee

Conflict of Intrests: None declared.

Ethical Approval: Ethical approval has been approved by IRB in Hallym University Sacred Heart Hospital. The number of IRB is HALLYM 2018-03-029.
Financial Disclosure: None of the authors have any financial interests relevant to the manuscript.

Funding/Support: Nothing to declare.

\section{References}

1. Murphey MD, Rhee JH, Lewis RB, Fanburg-Smith JC, Flemming DJ, Walker EA. Pigmented villonodular synovitis: Radiologicpathologic correlation. Radiographics. 2008;28(5):1493-518. doi: 10.1148/rg.285085134. [PubMed: 18794322].

2. Jo VY, Fletcher CD. WHO classification of soft tissue tumours: An update based on the 2013 (4th) edition. Pathology. 2014;46(2):95-104. doi: 10.1097/PAT.0000000000000050. [PubMed: 24378391].

3. Park JH, Ro KH, Lee DH. Localized nodular synovitis of the infrapatellar fat pad. Indian J Orthop. 2013;47(3):313-6. doi: 10.4103/00195413.111514. [PubMed: 23798766]. [PubMed Central: PMC3687912].

4. Cheng KK, Chan CH, Yip SL, Chan SK, Chow HL. Localized nodular synovitis: An uncommon cause of knee pain. Hong Kong J Radiol. 2013;16:296-300. doi: 10.12809/hkjr1312128.

5. Fukui K, Kaneuji A, Kinoshita E, Numata Y, Nojima T, Matsumoto T. Localized pigmented villonodular synovitis of the hip: Suddenonset pain caused by torsion of the tumor pedicle. Case Rep Orthop. 2013;2013:862935. doi: 10.1155/2013/862935. [PubMed: 24324906]. [PubMed Central: PMC3844195].

6. Howie CR, Smith GD, Christie J, Gregg PJ. Torsion of localised pigmented villonodular synovitis of the knee. J Bone Joint Surg Br. 1985;67(4):564-6. doi: 10.1302/0301-620X.67B4.4030851. [PubMed: 4030851].

7. Huang GS, Lee CH, Chan WP, Chen CY, Yu JS, Resnick D. Localized nodular synovitis of the knee: MR imaging appearance and clinical correlates in 21 patients. AJR Am J Roentgenol. 2003;181(2):539-43. doi: 10.2214/ajr.181.2.1810539. [PubMed:12876042].

8. Gouin F, Noailles T. Localized and diffuse forms of tenosynovial giant cell tumor (formerly giant cell tumor of the tendon sheath and pigmented villonodular synovitis). Orthop Traumatol Surg Res. 2017;103(1S):S91-7. doi: 10.1016/j.otsr.2016.11.002. [PubMed: 28057477].

9. Sharma H, Rana B, Mahendra A, Jane MJ, Reid R. Outcome of 17 pigmented villonodular synovitis (PVNS) of the knee at 6 years mean follow-up. Knee. 2007;14(5):390-4. doi: 10.1016/j.knee.2007.05.007. [PubMed: 17600720].

10. Lin J, Jacobson JA, Jamadar DA, Ellis JH. Pigmented villonodular synovitis and related lesions: The spectrum of imaging findings. AJR Am J Roentgenol. 1999;172(1):191-7. doi: 10.2214/ajr.172.1.9888766. [PubMed: 9888766].

11. Kim HS, Kwon JW, Ahn JH, Chang MJ, Cho EY. Localized tenosynovial giant cell tumor in both knee joints. Skeletal Radiol. 2010;39(9):923-6. doi: 10.1007/s00256-010-0910-8. [PubMed: 20354849].

12. Lee SY, Jee WH, Jung JY, Park MY, Kim SK, Jung CK, et al. Differentiation of malignant from benign soft tissue tumours: Use of additive qualitative and quantitative diffusion-weighted MR imaging to standard MRimaging at 3.0 T. Eur Radiol.2016;26(3):743-54. doi:10.1007/s00330015-3878-x. [PubMed: 26080796].

13. Jeon JY, Chung HW, Lee MH, Lee SH, Shin MJ. Usefulness of diffusionweighted MR imaging for differentiating between benign and malignant superficial soft tissue tumours and tumour-like lesions. $\mathrm{Br} J$ Radiol. 2016;89(1060):20150929. doi: 10.1259/bjr.20150929. [PubMed: 26892266]. [PubMed Central: PMC4846217].

14. Pekcevik Y, Kahya MO, Kaya A. Characterization of soft tissue tumors by diffusion-weighted imaging. Iran J Radiol. 2015;12(3). e15478. doi: 10.5812/iranjradiol.15478v2. [PubMed: 26557269]. [PubMed Central: PMC4632135]. 